\title{
Mitochondrial NADP(H) generation is essential for proline biosynthesis
}

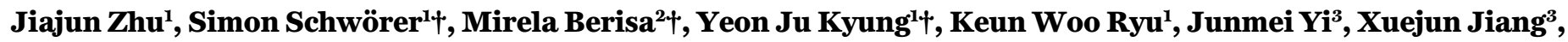 \\ Justin R. Cross ${ }^{2}$, Craig B. Thompson ${ }^{1 *}$
}

1Department of Cancer Biology and Genetics, Memorial Sloan Kettering Cancer Center, New York, NY 10065, USA. ${ }^{2}$ The Donald B. and Catherine C. Marron Cancer

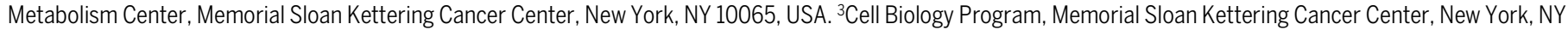
10065, USA.

†These authors contributed equally to this work.

*Corresponding author. Email: thompsonc@mskcc.org

The coenzyme nicotinamide adenine dinucleotide phosphate $\left(\right.$ NADP $\left.^{+}\right)$and its reduced form (NADPH) regulate reductive metabolism in a subcellularly compartmentalized manner. Mitochondrial NADP(H) production depends on the phosphorylation of $N A D(H)$ by NAD kinase 2 (NADK2). Deletion of NADK2 in human cell lines did not alter mitochondrial folate pathway activity, tricarboxylic acid cycle activity, or mitochondrial oxidative stress, but led to impaired cell proliferation in minimal medium. This growth defect was rescued by proline supplementation. NADK2-mediated mitochondrial NADP(H) generation was required for the reduction of glutamate and hence proline biosynthesis. Furthermore, mitochondrial $\operatorname{NADP}(\mathrm{H})$ availability determined the production of collagen proteins by cells of mesenchymal lineage. Thus, a primary function of the mitochondrial $\operatorname{NADP}(\mathrm{H})$ pool is to support proline biosynthesis for use in cytosolic protein synthesis.

Mammalian cells depend on the inter-conversion of nicotinamide adenine dinucleotide phosphate molecules between the oxidized $\left(\mathrm{NADP}^{+}\right)$and reduced $(\mathrm{NADPH})$ forms to support reductive biosynthesis and to maintain cellular antioxidant defense. $\mathrm{NADP}^{+}$and $\mathrm{NADPH}$ molecules $[\mathrm{NADP}(\mathrm{H})$ hereafter] are unable to cross subcellular membranes $(1,2)$. As a result, cellular pools of $\mathrm{NADP}(\mathrm{H})$ are compartmentalized. In the cytosol, $\mathrm{NADP}(\mathrm{H})$ is derived from nicotinamide adenine dinucleotide $[\mathrm{NAD}(\mathrm{H})]$ by NAD kinase (NADK, referred to as NADK1 hereafter). Cytosolic NADPH acts as a substrate in fatty acid biosynthesis, and as the reducing equivalent required to regenerate reduced glutathione (GSH) and thioredoxin for antioxidant defense. Mitochondria host a number of biosynthetic activities critical for cellular metabolism but are also major sites for reactive oxygen species (ROS) generation. Mammalian mitochondrial NAD kinase (NADK2) converts $\mathrm{NAD}(\mathrm{H})$ to $\mathrm{NADP}(\mathrm{H})$ through phosphorylation (3).

Using subcellular fractionation, we confirmed that NADK2 purified in the membrane-associated fraction in cultured human cell lines (fig. S1, A to C). Mitochondria immunopurification (Mito-IP, $(4,5))$ from DLD1 cells following CRISPR-Cas9 deletion of NADK2 (fig. S1D) resulted in a metabolomic profile consistent with mitochondrial metabolism, and metabolites known to be excluded from the mitochondrial compartment were minimally detected (Fig. 1A; fig. S1, E to G; and table S1). We examined $\operatorname{NADP}(\mathrm{H})$ levels in immunopurified mitochondria using an adapted enzyme cycling assay (6). Although total $\mathrm{NADP}(\mathrm{H})$ abundance or $\mathrm{NADP}^{+}$to NADPH ratio were not changed at whole cell level upon NADK2 loss as previously reported $(6,7)$, mitochondrial $\operatorname{NADP}(\mathrm{H})$ abundance was reduced by more than $80 \%$ $(P<0.001)$ in NADK2 knockout cells (Fig. 1, B and C, and fig. $\mathrm{S} 1, \mathrm{H}$ to $\mathrm{J})$. $\mathrm{NAD}(\mathrm{H})$ abundance or $\mathrm{NAD}^{+}$to $\mathrm{NADH}$ ratio were not altered by $N A D K 2$ knockout in whole cells or in mitochondria (fig. S1, $\mathrm{K}$ to $\mathrm{N}$ ).

Oncogenic mutant forms of isocitrate dehydrogenase 1 (IDH1) and IDH2 require cytosolic and mitochondrial $\mathrm{NADPH}$, respectively, to produce 2-hydroxyglutarate (2HG) from $\alpha$-ketoglutarate $(\alpha \mathrm{KG})(8)$ (fig. S1O). We deleted the NADK2 gene in chondrosarcoma cell lines that had either an endogenous IDH1 R132 mutation (JJ012) or IDH2 R172 mutation (CS1) (Fig. 1D). Loss of NADK2 resulted in reduced 2HG abundance $(P<0.001)$ in CS1 cells, but not in JJ012 cells (Fig. 1, E and F). We further subjected control and NADK2deleted CS1 cells to a xenograft tumor assay in vivo and observed similarly decreased $2 \mathrm{HG}$ abundance in tumors formed by NADK2 knockout cells (Fig. 1G). These results confirmed that NADK2 is required to maintain the mitochondrial $\operatorname{NADP}(\mathrm{H})$ pool.

Methylenetetrahydrofolate dehydrogenase 2 (MTHFD2) and MTHFD2-like (MTHFD2L) use either $\mathrm{NAD}^{+}$or $\mathrm{NADP}^{+}$as electron acceptors in the mitochondrial folate pathway. 
Using $\left[2,3,3-{ }^{2} \mathrm{H} 3\right]$ serine isotope tracing, cells lacking MTHFD2 or serine hydroxymethyltransferase 2 (SHMT2) both displayed an increase in doubly labeled thymidine triphosphate (TTP M+2) when compared to control cells (Fig. 2, A to C, and fig. S2, A and B), suggesting decreased mitochondrial folate pathway activity and increased cytosolic serine catabolism, as previously reported $(9,10)$. By contrast, cells lacking $N A D K 2$ maintained the fraction of singly labeled (TTP $\mathrm{M}+1$ ) derived from $\left[2,3,3{ }^{2} \mathrm{H} 3\right]$ serine (Fig. 2 , A to $\mathrm{C}$, and fig. S2, A and B), indicating the mitochondrial folate pathway is not disrupted by NADK2 loss.

We performed isotope tracing experiments with uniformly labeled $\left[\mathrm{U}^{-13} \mathrm{C}\right]$ glucose or $\left[\mathrm{U}-{ }^{13} \mathrm{C}\right]$ glutamine comparing control and NADK2-deleted cells to analyze tricarboxylic acid (TCA) cycle activity. We did not observe consistent changes in the TCA cycle intermediates derived from either glucose or glutamine (Fig. 2, D to G, and fig. S2, C to V). In addition, NADK2-deletion did not lead to changes in the mitochondrial basal oxygen consumption rate or uncoupled electron transport chain activity (fig. S2, W to Y).

Mitochondria are major sites of ROS generation in cells (11), and depletion of mitochondrial $\mathrm{NADP}(\mathrm{H})$ is thought to lead to oxidative stress. However, in all cell types that we tested, cells lacking NADK2 did not display increased cellular ROS or mitochondrial superoxide (MitoSox) abundance (Fig. $2 \mathrm{H}$ and fig. S3, A to G). We used mitochondria-targeted redoxsensitive green fluorescence protein (roGFP2) constructs coupled to the yeast peroxidase Orp1 or human glutaredoxin-1 (Grx1) $(12,13)$, and measured similar amounts of mitochondrial $\mathrm{H}_{2} \mathrm{O}_{2}$ or GSH oxidation, respectively, in control and NADK2 knockout cells (Fig. 2I and fig. S3, $\mathrm{H}$ to J). Treatment with MitoParaquat (MitoPQ) increased the expression of enzymes involved in GSH synthesis to a similar extent in cells lacking $N A D K 2$ as that in the control cells (14) (fig. S3, K and L). In agreement, loss of NADK2 did not alter cellular or mitochondrial GSH abundance or the ratio of GSH to its oxidized form GSSG (GSH/GSSG) (fig. S3, M to P). [U${ }^{13} \mathrm{C}$ ]glutamine tracing revealed no significant changes in the fraction of GSH or GSSG derived from glutamine upon NADK2 loss (fig. S3, Q and R). These results are consistent with the cytosolic $\operatorname{NADP}(\mathrm{H})$ pool, but not mitochondrial $\mathrm{NADP}(\mathrm{H})$, being critical for maintaining cellular GSH levels to prevent oxidative damage (7). Glutathione reductase (GSR) expression was absent in the mitochondrial fraction (Fig. 2J), thus the NADPH-dependent GSH reduction appears not to take place in mitochondria.

Hyper-oxidation of peroxiredoxins $\left(\mathrm{PRXs}^{-\mathrm{SO}_{3}}\right)$ indicates oxidative stress of the cellular thioredoxin system. We observed similar amounts of mitochondrial (PRX3), as well as cytosolic and nuclear (PRX1 and PRX2) peroxiredoxin oxidation, when comparing cells lacking $N A D K 2$ with control cells (Fig. 2K and fig. S3, S and T). Cellular and mitochondrial oxidative stress can lead to ferroptotic cell death $(15,16)$. When treated with Erastin or RSL3, chemicals that induce ferroptosis, cells lacking NADK2 showed no increase in cell death (Fig. 2L and fig. S3U). Similarly, Nadk2 knockout did not increase sensitivity to ferroptosis in contact-inhibited, non-proliferative mouse embryonic fibroblasts (MEFs) (fig. $\mathrm{S} 3, \mathrm{~V}$ and $\mathrm{W}$ ). Thus, loss of NADK2, and depletion of mitochondrial $\operatorname{NADP}(\mathrm{H})$, did not increase oxidative stress under the experimental conditions we examined, although it remains possible that mitochondrial $\mathrm{NADP}(\mathrm{H})$ generation might play a role in antioxidant defense in response to other physiological perturbations.

We observed that proliferation of cells lacking $N A D K 2$ was not perturbed compared to that of control cells when cultured in a nutrient rich medium (DMEM/F12) (fig. S4, A to D). However, our studies of IDH2-mutant cells indicated that NADK2 could have a role in NADPH-dependent biosynthesis (Fig. 1, F and G). To test whether mitochondrial $\operatorname{NADP}(\mathrm{H})$ supports biosynthetic reactions in general, we subjected control and NADK2 knockout cells to culture medium composed of minimal essential nutrients (DMEM) and found that the growth of NADK2-deleted cells was compromised (fig. S4, A to $\mathrm{D})$. Apparently, mitochondrial $\mathrm{NADP}(\mathrm{H})$ promotes the synthesis of one or more nutrients required to sustain cell proliferation.

Growth of cells lacking NADK2 was restored in DMEM by supplementing non-essential amino acids (NEAAs), but not by other nutrients present in DMEM/F12 (Fig. 3A and fig. S4, $\mathrm{E}$ and $\mathrm{F})$. Supplementing individual amino acids revealed that proline was both necessary and sufficient to restore proliferation of $N A D K 2$ knockout cells in DMEM (Fig. 3B and fig. $\mathrm{S} 4, \mathrm{G}$ to $\mathrm{J})$. In agreement, cells lacking $N A D K 2$ showed reduced intracellular proline abundance (Fig. 3C). Similar results were obtained under hypoxia $\left(0.5 \% \mathrm{O}_{2}\right)$ (fig. $\mathrm{S} 4, \mathrm{~K}$ to $\left.\mathrm{M}\right)$. To validate that the proline-dependent growth phenotype was the result of NADK2 loss, we introduced NADK2 cDNA resistant to CRISPR-Cas9 mediated genome editing into the NADK2 knockout cells, which restored both intracellular proline abundance and cell growth (Fig. 3, D to F, and fig. S5, A to $\mathrm{C})$. Similar results were observed when the yeast mitochondrial NAD $(\mathrm{H})$ kinase, POS5 (17), was reconstituted in NADK2deficient cells (Fig. 3, G to I, and fig. S5, D to F).

We performed metabolite profiling of cells lacking NADK2 cultured in DMEM, and confirmed the depletion of intracellular proline, while amounts of many other amino acids were slightly increased (Fig. 4A and fig. S6, A and B). Loss of NADK2 also reduced proline abundance in non-proliferating (contact-inhibited) MEFs (fig. S6, C and D). By contrast, loss of cytosolic NADK1 did not decrease proline abundance (fig. S6, E and F). Likewise, the oxygen-dependent NADPH oxidase, TPNOX (18), reduced proline amounts when expressed in mitochondria (mitoTPNOX) but not in cytosol 
(cytoTPNOX) (fig. S6, G to J). To extend these observations, we examined the consumption of nutrients from the prolinecontaining DMEM/F12 medium. While we observed net proline accumulation in medium conditioned by control cells, proline was consumed by cells lacking $N A D K 2$ (Fig. 4, B and $\mathrm{C}$, and fig. S7, A to D). In addition, glutamate accumulation was found in medium conditioned by cells lacking NADK2 (Fig. 4, B and D, and fig. S7, A, B, E, and F), which might result from compensatory accumulation of carbon and nitrogen in the form of glutamate instead of proline. We performed similar analyses in xenograft tumors formed by CS1 cells (Fig. 1). We found that across a panel of amino acids, proline amount was reduced in tumors formed by CS1 cells lacking $N A D K 2$ (Fig. 4E and fig. S7G), which correlated with a slower growth rate of these tumors compared to those formed by control cells (fig. S7H). Mice grafted with control or NADK2 knockout cells displayed similar plasma levels of proline as well as other amino acids at the time of tumor resection (fig. S7I). Thus, loss of NADK2, and the consequent depletion of mitochondrial $\mathrm{NADP}(\mathrm{H})$, results in proline auxotrophy.

Proline biosynthesis takes place in the mitochondria, where glutamine-derived glutamate is converted to pyrroline5-carboxylate (P5C) by pyrroline-5-carboxylate synthase (P5CS). P5C is further reduced to proline by mitochondrial pyrroline-5-carboxylate reductases (PYCR1 and PYCR2) (Fig. $4 \mathrm{~F})$. $\left[\mathrm{U}-{ }^{13} \mathrm{C}\right]$ glutamine tracing revealed that most cellular glutamate and proline were derived from glutamine, and that glutamine-derived proline was reduced upon NADK2 loss (Fig. 4, G and H, and fig. S8, A and B). By contrast, proline abundance was not perturbed when the cytosolic pyrroline5-carboxylate reductase (PYCRL) was deleted (fig. S8, C and D).

P5CS is an NADPH-dependent enzyme, whereas PYCR1 and PYCR2 have higher affinity for NADH than for NADPH (19-21). To test if loss of NADK2 impairs conversion of glutamate to P5C by P5CS, we took advantage of the fact that cellular P5C is in equilibrium with glutamate-5-semialdhyde (GSA), which can be diverted to produce ornithine for polyamine biosynthesis (Fig. 4F). Intracellular arginine can also contribute to ornithine and polyamines. Isotope tracing using $\left[\mathrm{U}-{ }^{13} \mathrm{C}\right]$ glutamine and $\left[\mathrm{U}_{-}{ }^{13} \mathrm{C}\right]$ arginine allowed us to assess the relative contribution of these pathways to polyamine production (fig. S8E). The fraction of ornithine and putrescine derived from $\left[\mathrm{U}^{13} \mathrm{C}\right]$ glutamine decreased in cells lacking $N A D K 2$, indicating that P5CS flux from glutamate to $\mathrm{P} 5 \mathrm{C}$ and GSA was diminished (Fig. 4, I and J). This also resulted in a reciprocal increase in the proportional contribution of arginine to ornithine and putrescine (fig. S8, F to I). Because ornithine transcarbamylase expression is restricted to the liver and small intestine, loss of NADK2 did not change glutamine or arginine contribution to cellular citrulline (fig. S8, J and $\mathrm{K})$. Thus, loss of NADK2 and the resulting decrease in mitochondrial NADP(H) blocks the reduction of glutamate to $\mathrm{P} 5 \mathrm{C}$ required for proline biosynthesis.

Incorporation of the proline pyrrolidine ring slows protein translation $(22,23)$, but endows proline-containing polypeptides with conformational rigidity. As a result, proline and its post-translationally modified form, hydroxyproline, are abundant in collagen proteins (24), so a consequence of decreased mitochondrial $\mathrm{NADP}(\mathrm{H})$ generation could be impaired collagen production. Cultured mouse fibroblasts lacking Nadk2 had decreased expression of collagen when grown in DMEM (Fig. 4K and fig. S9A). These cells accumulated activating transcription factor 4 (ATF4), indicative of amino acid shortage. Addition of $300 \mu \mathrm{M}$ proline to the culture medium restored collagen expression and blunted ATF4 accumulation in cells lacking Nadk2 (Fig. $4 \mathrm{~K}$ and fig. S9, A and B). Similar results were obtained in osteosarcoma and chondrosarcoma cells that produce collagens (fig. S9, C and D). Fibroblasts lacking Nadk2 showed decreased collagen secretion, which was rescued by proline supplementation to the medium (Fig. 4, L and M). In patients with idiopathic pulmonary fibrosis (IPF) (25), higher $N A D K 2$ expression in the lung correlated with lower forced vital capacity $(\mathrm{FVC})(P=0.007)$ and diffusion capacity for carbon monoxide (DLCO) $(P=$ 0.015), parameters that measure maximum air exhalation and the ability of lung to transfer air into the blood, respectively (Fig. 4, N and O). Similarly, IPF patients with both high $N A D K 2$ and high P5CS expression in the lung had reduced FVC and DLCO values compared to those with low NADK2 and low P5CS expression (fig. S9, E and F). Thus, increased expression of $N A D K 2$ correlated with enhanced fibrotic diseases characterized by excessive collagen deposition.

These findings provide insights into the regulation of intracellular metabolism. In endosymbiosis with the host cell, mitochondria produce $\operatorname{NADP}(\mathrm{H})$ that supplies biosynthetic precursors to their host and appear not to use the NADP $(\mathrm{H})$ for antioxidant defense in support of their own homeostasis. Compartmentalization of cellular metabolism thus has important roles in eukaryotic cells beyond the well-known collaborative production of ATP.

\section{REFERENCES AND NOTES}

1. R. P. Goodman, S. E. Calvo, V. K. Mootha, Spatiotemporal compartmentalization of hepatic NADH and NADPH metabolism. J. Biol. Chem. 293, 7508-7516 (2018). doi:10.1074/jbc.TM117.000258 Medline

2. C. A. Lewis, S. J. Parker, B. P. Fiske, D. McCloskey, D. Y. Gui, C. R. Green, N. I. Vokes, A. M. Feist, M. G. Vander Heiden, C. M. Metallo, Tracing compartmentalized NADPH metabolism in the cytosol and mitochondria of mammalian cells. Mol. Cell 55, 253-263 (2014). doi:10.1016/j.molcel.2014.05.008 Medline

3. K. Ohashi, S. Kawai, K. Murata, Identification and characterization of a human mitochondrial NAD kinase. Nat. Commun. 3, 1248 (2012). doi:10.1038/ncomms2262 Medline

4. W. W. Chen, E. Freinkman, D. M. Sabatini, Rapid immunopurification of mitochondria for metabolite profiling and absolute quantification of matrix metabolites. Nat. Protoc. 12, 2215-2231 (2017). doi:10.1038/nprot.2017.104 Medline 
5. W. W. Chen, E. Freinkman, T. Wang, K. Birsoy, D. M. Sabatini, Absolute Quantification of Matrix Metabolites Reveals the Dynamics of Mitochondrial Metabolism. Cell 166, 1324-1337.el1 (2016). doi:10.1016/i.cell.2016.07.040 Medline

6. G. Hoxhaj, I. Ben-Sahra, S. E. Lockwood, R. C. Timson, V. Byles, G. T. Henning, P. Gao, L. M. Selfors, J. M. Asara, B. D. Manning, Direct stimulation of NADP+ synthesis through Akt-mediated phosphorylation of NAD kinase. Science 363 , 1088-1092 (2019). doi:10.1126/science.aau3903 Medline

7. C. C. Ding, J. Rose, T. Sun, J. Wu, P.-H. Chen, C.-C. Lin, W.-H. Yang, K.-Y. Chen, H. Lee, E. Xu, S. Tian, J. Akinwuntan, J. Zhao, Z. Guan, P. Zhou, J.-T. Chi, MESH1 is a cytosolic NADPH phosphatase that regulates ferroptosis. Nat. Metab. 2, 270-277 (2020). doi:10.1038/s42255-020-0181-1 Medline

8. A. H. Shih, O. Abdel-Wahab, J. P. Patel, R. L. Levine, The role of mutations in epigenetic regulators in myeloid malignancies. Nat. Rev. Cancer 12, 599-612 (2012). doi:10.1038/nrc3343 Medline

9. G. S. Ducker, L. Chen, R. J. Morscher, J. M. Ghergurovich, M. Esposito, X. Teng, Y. Kang, J. D. Rabinowitz, Reversal of Cytosolic One-Carbon Flux Compensates for Loss of the Mitochondrial Folate Pathway. Cell Metab. 23, 1140-1153 (2016). doi:10.1016/i.cmet.2016.04.016 Medline

10. N. Kory, G. A. Wyant, G. Prakash, J. uit de Bos, F. Bottanelli, M. E. Pacold, S. H. Chan, C. A. Lewis, T. Wang, H. R. Keys, Y. E. Guo, D. M. Sabatini, SFXN1 is a mitochondrial serine transporter required for one-carbon metabolism. Science 362, eaat9528 (2018). doi:10.1126/science.aat9528 Medline

11. H. Kong, N. S. Chandel, Regulation of redox balance in cancer and T cells. J. Biol. Chem. 293, 7499-7507 (2018). doi:10.1074/ibc. TM117.000257 Medline

12. M. Gutscher, A.-L. Pauleau, L. Marty, T. Brach, G. H. Wabnitz, Y. Samstag, A. J. Meyer, T. P. Dick, Real-time imaging of the intracellular glutathione redox potential. Nat. Methods 5, 553-559 (2008). doi:10.1038/nmeth.1212 Medline

13. M. Gutscher, M. C. Sobotta, G. H. Wabnitz, S. Ballikaya, A. J. Meyer, Y. Samstag, T. P. Dick, Proximity-based protein thiol oxidation by $\mathrm{H}_{2} \mathrm{O}_{2}$-scavenging peroxidases. J. Biol. Chem. 284, 31532-31540 (2009). doi:10.1074/jbc.M109.059246 Medline

14. E. L. Robb, J. M. Gawel, D. Aksentijević, H. M. Cochemé, T. S. Stewart, M. M. Shchepinova, H. Qiang, T. A. Prime, T. P. Bright, A. M. James, M. J. Shattock, H. M. Senn, R. C. Hartley, M. P. Murphy, Selective superoxide generation within mitochondria by the targeted redox cycler MitoParaquat. Free Radic. Biol. Med. 89, 883-894 (2015). doi:10.1016/j.freeradbiomed.2015.08.021 Medline

15. M. Gao, J. Yi, J. Zhu, A. M. Minikes, P. Monian, C. B. Thompson, X. Jiang, Role of Mitochondria in Ferroptosis. Mol. Cell 73, 354-363.e3 (2019). doi:10.1016/i.molcel.2018.10.042 Medline

16. B. R. Stockwell, J. P. Friedmann Angeli, H. Bayir, A. I. Bush, M. Conrad, S. J. Dixon, S. Fulda, S. Gascón, S. K. Hatzios, V. E. Kagan, K. Noel, X. Jiang, A. Linkermann, M. E. Murphy, M. Overholtzer, A. Oyagi, G. C. Pagnussat, J. Park, Q. Ran, C. S. Rosenfeld, K. Salnikow, D. Tang, F. M. Torti, S. V. Torti, S. Toyokuni, K. A. Woerpel, D. D. Zhang, Ferroptosis: A Regulated Cell Death Nexus Linking Metabolism, Redox Biology, and Disease. Cell 171, 273-285 (2017). doi:10.1016/i.cell.2017.09.021 Medline

17. C. E. Outten, V. C. Culotta, A novel NADH kinase is the mitochondrial source of NADPH in Saccharomyces cerevisiae. EMBO J. 22, 2015-2024 (2003). doi:10.1093/emboj/cdg211 Medline

18. V. Cracan, D. V. Titov, H. Shen, Z. Grabarek, V. K. Mootha, A genetically encoded tool for manipulation of NADP+/NADPH in living cells. Nat. Chem. Biol. 13, 10881095 (2017). doi:10.1038/nchembio.2454 Medline

19. J. De Ingeniis, B. Ratnikov, A. D. Richardson, D. A. Scott, P. Aza-Blanc, S. K. De, M. Kazanov, M. Pellecchia, Z. Ronai, A. L. Osterman, J. W. Smith, Functional specialization in proline biosynthesis of melanoma. PLOS ONE 7, e45190 (2012). doi:10.1371/journal.pone.0045190 Medline

20. J. J. Kramer, R. C. Gooding, M. E. Jones, A radiochemical assay for a NADP+ specific $Y_{\text {-glutamate semialdehyde dehydrogenase extracted from mitochondrial }}$ membrane of rat intestinal epithelial cells. Anal. Biochem. 168, 380-386 (1988). doi:10.1016/0003-2697(88)90333-8 Medline

21. J. M. Phang, Proline Metabolism in Cell Regulation and Cancer Biology: Recent Advances and Hypotheses. Antioxid. Redox Signal. 30, 635-649 (2019). doi:10.1089/ars.2017.7350 Medline

22. M. Y. Pavlov, R. E. Watts, Z. Tan, V. W. Cornish, M. Ehrenberg, A. C. Forster, Slow peptide bond formation by proline and other $\mathrm{N}$-alkylamino acids in translation.
Proc. Natl. Acad. Sci. U.S.A. 106, 50-54 (2009). doi:10.1073/pnas.0809211106 Medline

23. F. Loayza-Puch, K. Rooijers, L. C. M. Buil, J. Zijlstra, J. F. Oude Vrielink, R. Lopes, A. P. Ugalde, P. van Breugel, I. Hofland, J. Wesseling, O. van Tellingen, A. Bex, R. Agami, Tumour-specific proline vulnerability uncovered by differential ribosome codon reading. Nature 530, 490-494 (2016). doi:10.1038/nature16982 Medline

24. S. Schwörer, M. Berisa, S. Violante, W. Qin, J. Zhu, R. C. Hendrickson, J. R. Cross, C. B. Thompson, Proline biosynthesis is a vent for TGF $\beta$-induced mitochondrial redox stress. EMBO J. 39, e103334 (2020). doi:10.15252/embj.2019103334 Medline

25. I. V. Yang, C. D. Coldren, S. M. Leach, M. A. Seibold, E. Murphy, J. Lin, R. Rosen, A J. Neidermyer, D. F. McKean, S. D. Groshong, C. Cool, G. P. Cosgrove, D. A. Lynch, K. K. Brown, M. I. Schwarz, T. E. Fingerlin, D. A. Schwartz, Expression of ciliumassociated genes defines novel molecular subtypes of idiopathic pulmonary fibrosis. Thorax 68, 1114-1121 (2013). doi:10.1136/thoraxinl-2012-202943 Medline

26. P. S. Ward, C. Lu, J. R. Cross, O. Abdel-Wahab, R. L. Levine, G. K. Schwartz, C. B. Thompson, The potential for isocitrate dehydrogenase mutations to produce 2hydroxyglutarate depends on allele specificity and subcellular compartmentalization. J. Biol. Chem. 288, 3804-3815 (2013). doi:10.1074/ibc.M112.435495 Medline

27. L. Salamanca-Cardona, H. Shah, A. J. Poot, F. M. Correa, V. Di Gialleonardo, H. Lui, V. Z. Miloushev, K. L. Granlund, S. S. Tee, J. R. Cross, C. B. Thompson, K. R Keshari, In Vivo Imaging of Glutamine Metabolism to the Oncometabolite 2Hydroxyglutarate in IDH1/2 Mutant Tumors. Cell Metab. 26, 830-841.e3 (2017). doi:10.1016/i.cmet.2017.10.001 Medline

28. S. Baghirova, B. G. Hughes, M. J. Hendzel, R. Schulz, Sequential fractionation and isolation of subcellular proteins from tissue or cultured cells. MethodsX 2, 440445 (2015). doi:10.1016/i.mex.2015.11.001 Medline

\section{ACKNOWLEDGMENTS}

We thank members of the Thompson laboratory for critical discussions during manuscript preparation. We thank Elisa de Stanchina for assistance with tumor xenograft experiments, and Sara Violante for assistance with LC-MS experiments. Funding: J.Z. is supported by the Leukemia and Lymphoma Society (5460-18) and the $\mathrm{NCl}$ (1K99CA248711). S.S. is supported by the Human Frontier Science Program (LT000854/2018-L), the European Molecular Biology Organization (ALTF 467-2018), and the Alan and Sandra Gerry Metastasis and Tumor Ecosystems Center. K.W.R is supported by the Hunter Douglas Fellowship in Breast Cancer Research (13459) and the BRIA Postdoctoral Researcher Innovation Grant (18057). J.R.C. is supported by grants from the NIAID (R25 training grant Al140472-01A1) and the Donald B. and Catherine C. Marron Cancer Metabolism Center. X.J. is supported by grants from the NIH (R01CA204232 and R01CA166413). This work is supported by grants from the $\mathrm{NCl}$ (C.B.T.) and by the cancer center support grant (P30 CA008748) to Memorial Sloan Kettering Cancer Center. Author contributions: J.Z. and C.B.T. conceived the study. J.Z. performed most experiments and analyzed the data, with assistance from Y.J.K. S.S. performed some of the experiments in mouse fibroblasts, analyzed IPF datasets and optimized experimental methods used in this study. M.B. performed LC-MS measurements and analyses under the supervision of J.R.C. K.W.R. contributed key reagents and experimental methods. J.Y. and X.J. performed ferroptosis assays. J.Z. and C.B.T. interpreted the results and wrote the manuscript. All authors participated in discussing and finalizing the manuscript. Competing interests: C.B.T. is a founder of Agios Pharmaceuticals and a member of its scientific advisory board. He is also a former member of the Board of Directors and stockholder of Merck and Charles River Laboratories. He holds patents related to cellular metabolism. X.J. holds patents related to autophagy and cell death. Data and materials availability: All data are available in the manuscript or supplementary materials.

\section{SUPPLEMENTARY MATERIALS}

science.sciencemag.org/cgi/content/full/science.abd5491/DC1

Materials and Methods

Figs. S1 to S9 
Tables S1 and S2

References (26-28)

MDAR Reproducibility Checklist

27 June 2020; accepted 7 April 2021

Published online 22 April 2021

10.1126/science.abd5491 
NADK2 is required to maintain the mitochondrial NADP(H) pool
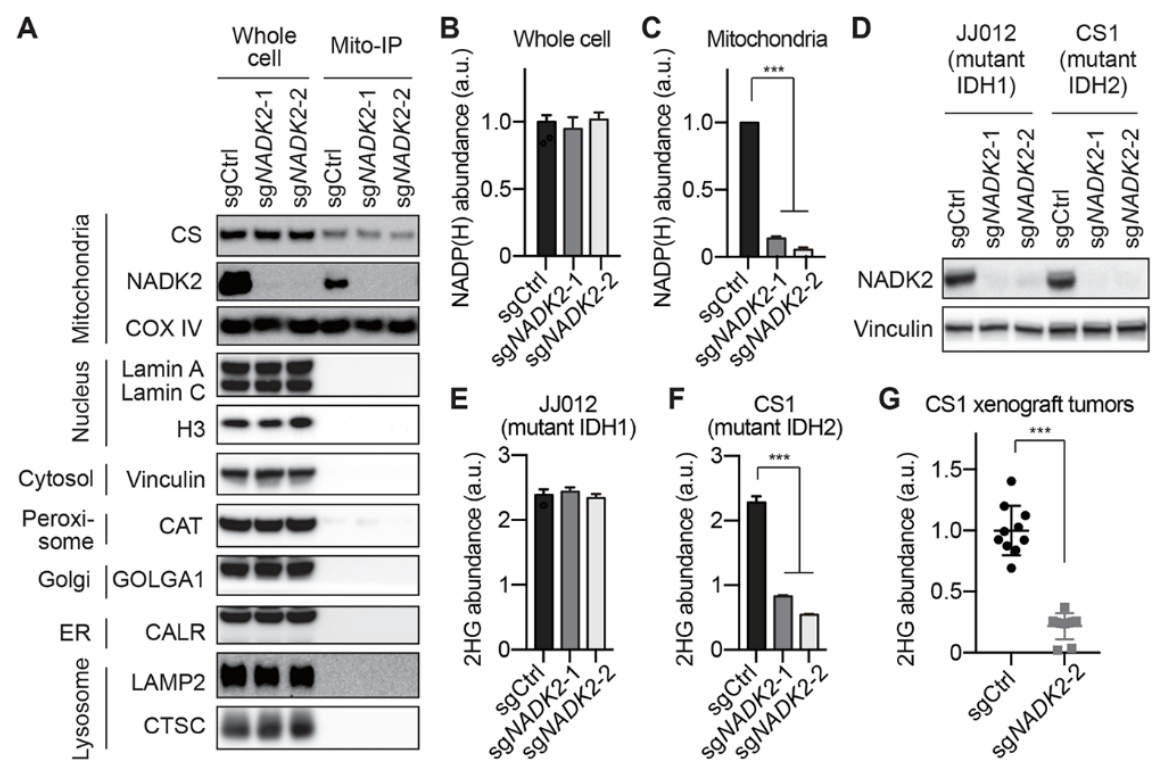

Fig. 1. NADK2 is required to maintain the mitochondrial NADP(H) pool. (A) DLD1 cells expressing HA-tagged OMP25 protein (DLD1OMP25HA) were engineered to express control guide RNA (sgCtrl) or two independent guide RNA sequences targeting NADK2 (sgNADK2-1 and sgNADK2-2), and were subjected to Western blot of whole cell or anti-HA immunopurified mitochondria (Mito-IP). (B and C) Colorimetric enzyme-based measurement of total $\operatorname{NADP}(H)$ abundance in $(B)$ whole cell or (C) immunopurified mitochondria of DLD1-OMP25HA cells with sgCtrl, sgNADK2-1, or sgNADK2-2, cultured in DMEM/F12 medium. (D) Western blot analysis of JJO12 (mutant IDH1) and CS1 (mutant IDH2) cells with sgCtrl, sgNADK2-1, or sgNADK2-2. (E and F) 2HG abundance measured by gas chromatography-mass spectrometry (GC-MS) in (E) JJO12 and (F) CS1 cells with sgCtrl, sgNADK2-1, or sgNADK2-2. (G) 2HG abundance measured by GC-MS in xenograft tumors formed by CS1 cells with sgCtrl or sgNADK2-2. Error bars in (B) represent mean $+\mathrm{SD}, \mathrm{n}$ $=6$; in $(C),(E)$ and $(F)$ represent mean $+S D, n=3$; in $(G)$ represent mean $\pm \mathrm{SD}, \mathrm{n}=10$. In $(\mathrm{C})$, one-way ANOVA was performed with matched measures. In (F), one-way ANOVA was performed. In (G), two-sided $t$ test was performed with Welch's correction. ${ }^{* * *} P<0.001$. 
Mitochondrial NADP(H) depletion has minimal effect on folate pathway and TCA cycle activity
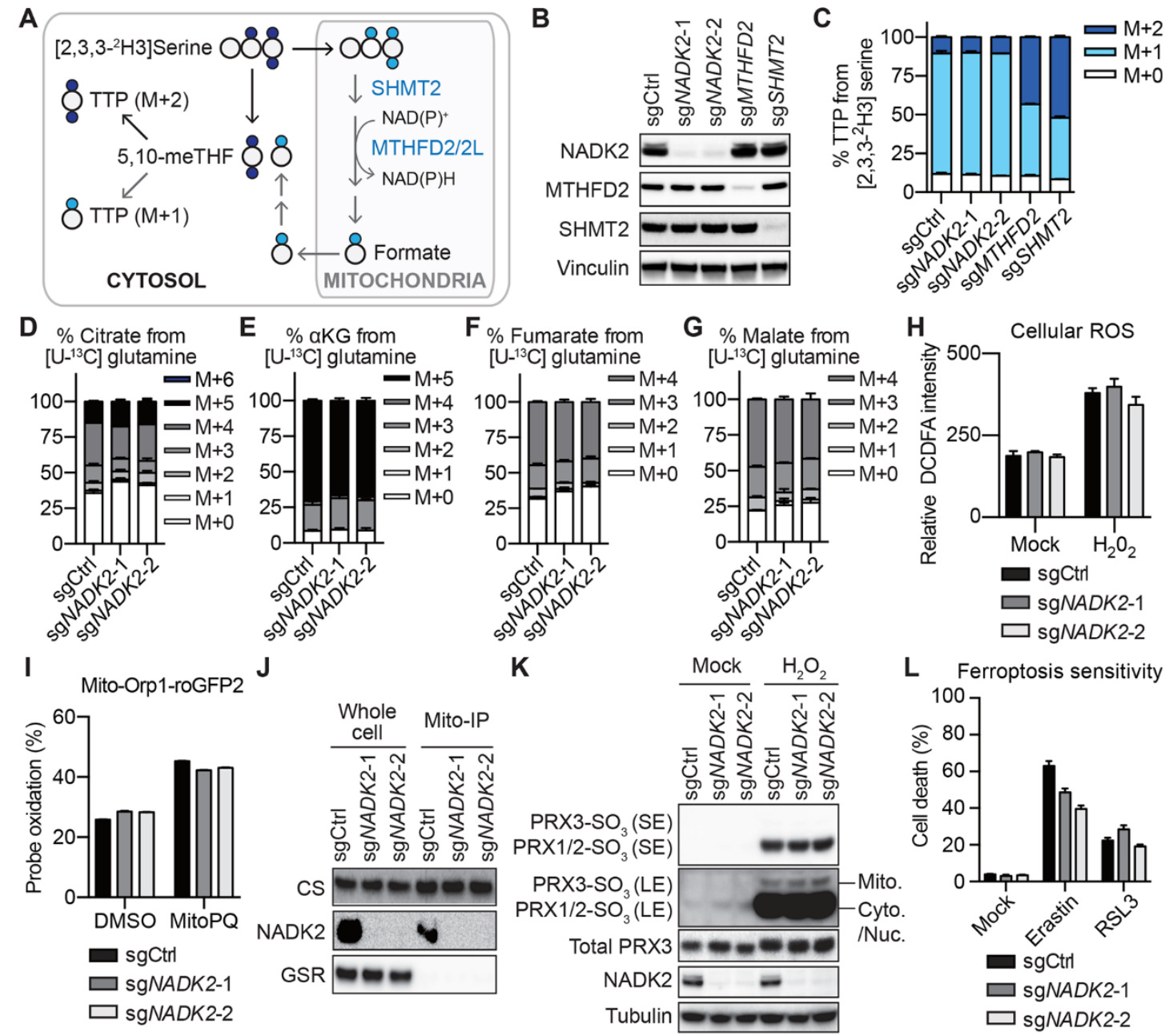

Fig. 2. Mitochondrial NADP $(H)$ depletion does not have significant effects on folate pathway, TCA cycle activity, or measures of oxidative stress. (A) Scheme of the tracing strategy, adapted from (9, 10). Catabolism of $\left[2,3,3-{ }^{2} \mathrm{H} 3\right]$ serine in the mitochondrial or cytosolic folate pathway produces singly or doubly deuterated thymidine triphosphate (TTP M+1 or TTP M+2), respectively. (B) Western blot of DLD1 cells with sgCtrl, sgNADK2-1, sgNADK2-2, sgMTHFD2, or sgSHMT2. (C) Isotopologue distribution of TTP measured by liquid chromatography-mass spectrometry (LC-MS) in DLD1 cells denoted in (B), cultured in [2,3,3-2 $\mathrm{H} 3]$ serine-containing medium for 8 hours. ( $D$ to $G$ ) Isotopologue distribution of the indicated metabolites measured by GC-MS in DLD1 cells with sgCtrl, sgNADK2-1, or sgNADK2-2, cultured in $\left[\mathrm{U}-{ }^{13} \mathrm{C}\right]$ glutamine-containing medium for 6 hours. $(\mathrm{H})$ Cellular ROS measured by CM$\mathrm{H}_{2}$ DCFDA in the indicated DLD1 cells, mock treated or treated with $150 \mu \mathrm{M} \mathrm{H}_{2} \mathrm{O}_{2}$ for 4 hours. (I) DLD1 cells expressing Mito-Orp1-roGFP2 and the indicated guide RNA were treated with vehicle (DMSO) or $100 \mu \mathrm{M}$ MitoPQ for 24 hours. Oxidation status was expressed as percentage of maximal oxidation which was determined by treating cells with $5 \mathrm{mM} \mathrm{H}_{2} \mathrm{O}_{2}$ for 5 min before harvest. (J) Western blot analysis of whole cell or immunopurified mitochondria of DLD1-OMP25HA cells expressing the indicated guide RNA (K) Western blot of the indicated DLD1 cells mock treated or treated with $500 \mu \mathrm{M} \mathrm{H}_{2} \mathrm{O}_{2}$ for 6 hours. SE, short exposure. LE, long exposure. (L) Ferroptosis sensitivity of the indicated DLD1 cells, measured as percentage cell death upon mock, Erastin $(5 \mu \mathrm{M})$ or RSL3 $(0.5 \mu \mathrm{M})$ treatment for 24 hours. All error bars in this figure represent mean $+S D, n=3$. 
Mitochondrial $\operatorname{NADP}(\mathrm{H})$ depletion results in proline auxotrophy
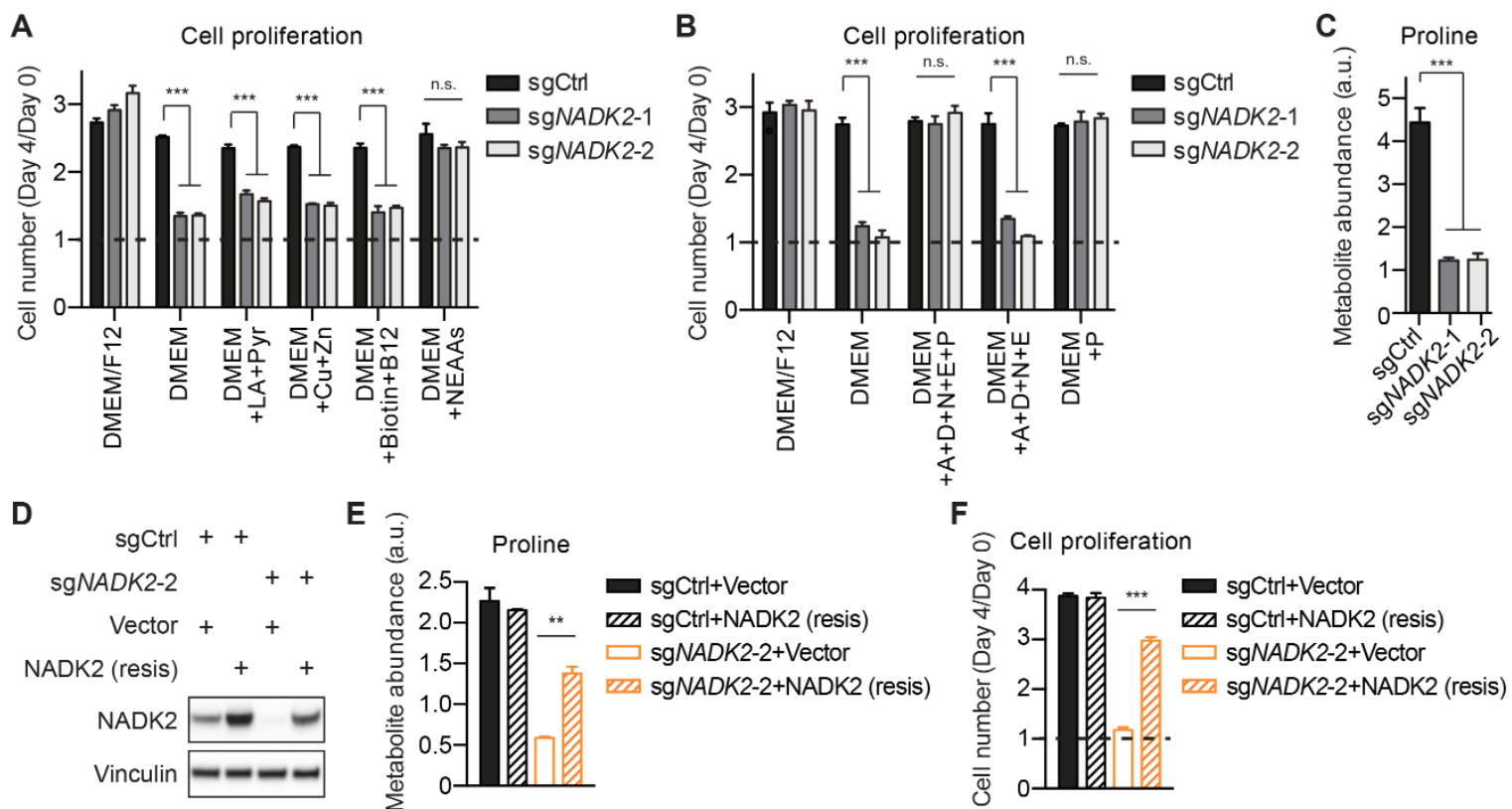

E

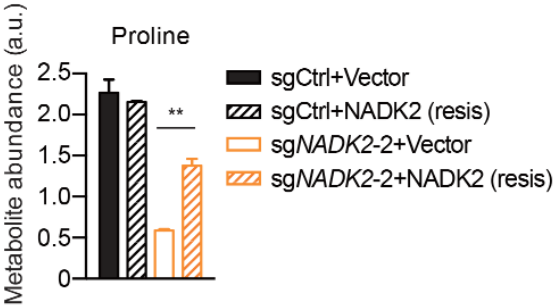

$\mathbf{F}$
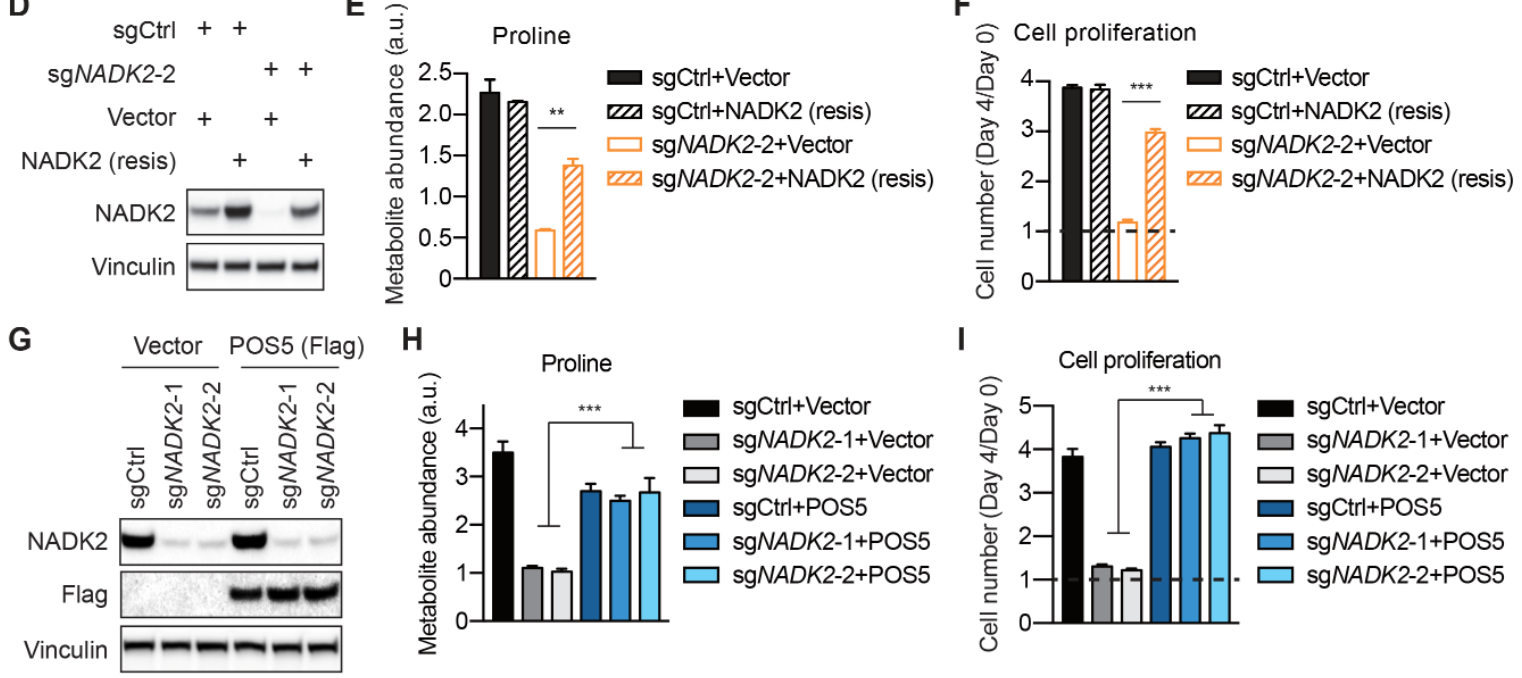

Fig. 3. Mitochondrial $\operatorname{NADP}(\mathrm{H})$ depletion results in proline auxotrophy. (A and $B$ ) Cell proliferation measured as cell number fold change (Day 4/Day 0) of T47D cells with sgCtrl, sgNADK2-1, or sgNADK2-2, cultured in the indicated medium and supplementation. LA, lipoic acid. Pyr, pyruvate. Cu, cupric sulfate. Zn, zinc sulfate. B12, vitamin B12. A, alanine. D, aspartate. $\mathrm{N}$, asparagine. E, glutamate. P, proline. All supplements are added at the concentrations present in DMEM/F12. (C) Proline abundance measured by GC-MS in the indicated T47D cells cultured in DMEM. (D to F) (D) Western blot, (E) proline abundance measured by GC-MS, and (F) cell proliferation of DMEM-cultured T47D cells with sgCtrl or sgNADK2-2 and ectopically expressing vector or NADK2 cDNA resistant to sgNADK2-2 mediated CRISPR-Cas9 genome editing. ( $G$ to I) $(\mathrm{G})$ Western blot, $(\mathrm{H})$ proline abundance measured by GC-MS, and (I) cell proliferation of DMEM-cultured T47D cells with sgCtrl, sgNADK2-1, or sgNADK2-2 and ectopically expressing vector or the POS5 CDNA. All error bars in this figure represent mean $+S D, n=3 . \ln (A),(B),(C)$, $(\mathrm{H})$ and $(\mathrm{I})$, one-way ANOVA was performed. In $(E)$ and $(F)$, two-sided $t$-test was performed with Welch's correction. ${ }^{*} P<0.01 ;{ }^{* *} P<0.001 ;$ n.s., $P>0.05$. 
. The mitochondrial $\operatorname{NADP}(\mathrm{H})$ pool is required to support proline biosynthesis and collagen production

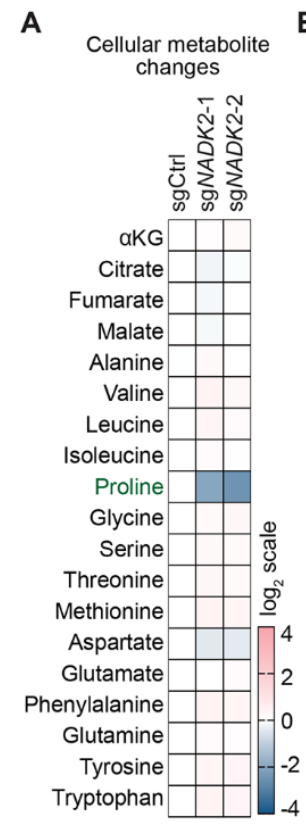

K

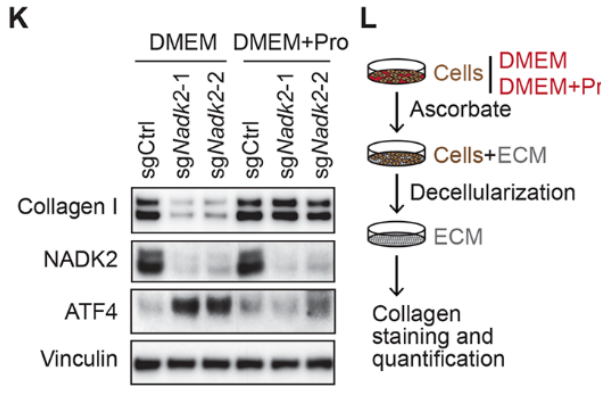

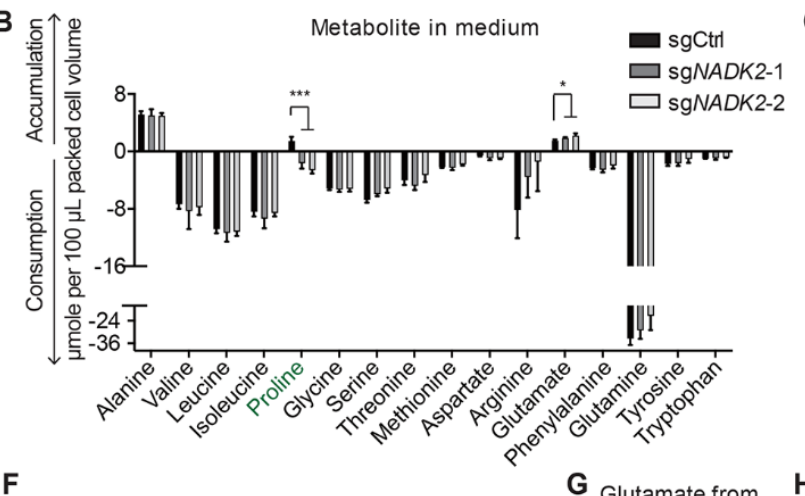

$\mathbf{F}$

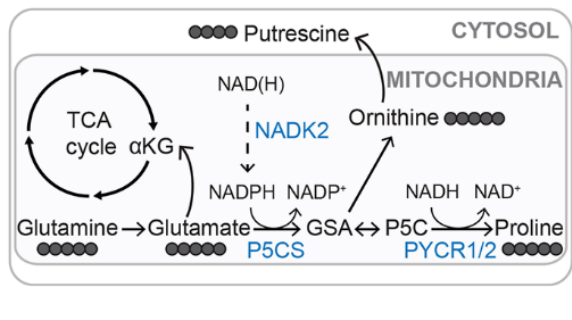

C

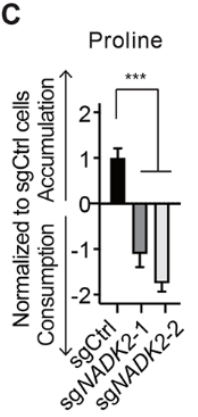

G Glutamate from
D $\quad E$

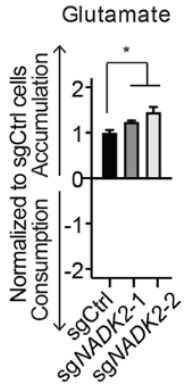

H Proline from I

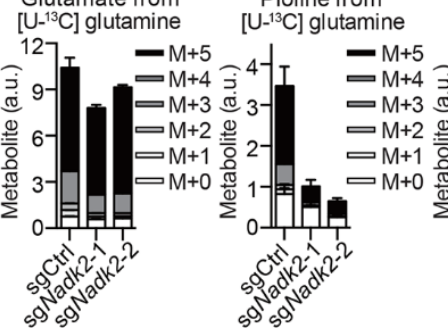

$\rightarrow \cos ^{\circ}$

$\cos ^{\circ} \mathrm{s}^{2}$

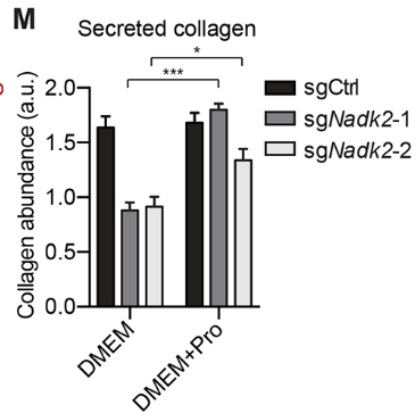

N

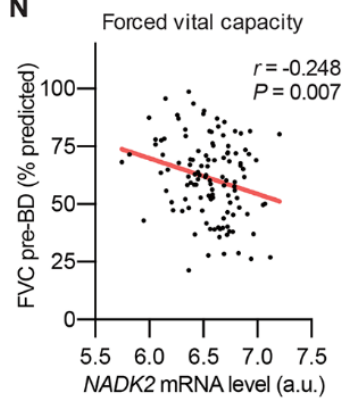

E Proline in CS1 xenograft tumors

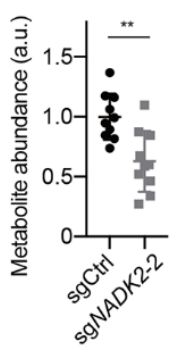

$J$ Putrescine from $\left[\mathrm{U}-{ }^{13} \mathrm{C}\right]$ glutamine
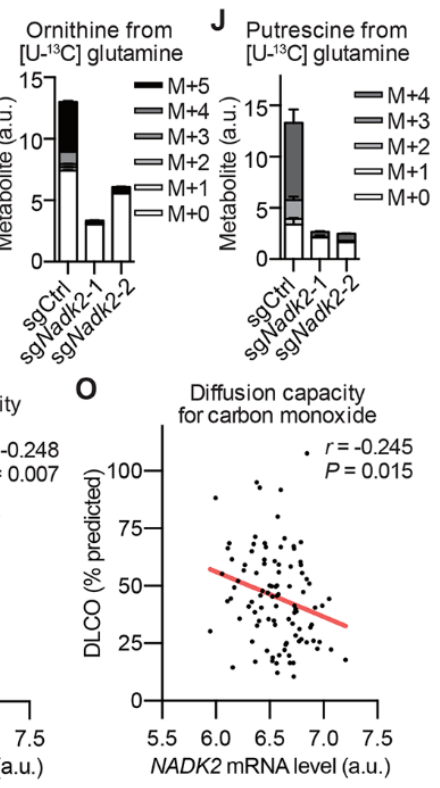

Fig. 4. The mitochondrial $\operatorname{NADP}(\mathrm{H})$ pool is required to support proline biosynthesis and collagen production. (A) Heatmap representing changes of metabolite levels measured by GC-MS in T47D cells with sgCtrl, sgNADK2-1, or sgNADK2-2 cultured in DMEM for 48 hours. The average of 3 biological replicates is shown. For each metabolite, values of sgNADK2-1 and sgNADK2-2 cells are shown as $\log _{2}$ (fold change) relative to the value of sgCtrl cells. (B) Changes of metabolite levels measured by GC-MS in DMEM/F12 medium used to culture T47D cells with sgCtrl, sgNADK2-1, or sgNADK2-2 for 48 hours. (C and D) (C) proline and (D) glutamate data from (B) re-plotted as normalized values to sgCtrl cells. (E) Proline abundance measured by GC-MS in xenograft tumors formed by CS1 cells with sgCtrl or sgNADK2-2. (F) Scheme of proline biosynthesis pathway in the mitochondria. ( $G$ to $J$ ) Relative total level and isotopologue distribution of the indicated metabolites measured by LC-MS in MEFs with sgCtrl, sgNadk2-1, or sgNadk2-2, cultured in DMEM containing $\left[\mathrm{U}-{ }^{13} \mathrm{C}\right]$ glutamine for 8 hours. (K) Western blot of the indicated MEFs, cultured in DMEM or DMEM supplemented with $300 \mu \mathrm{M}$ proline. (L) Scheme of ECM extraction and collagen staining in cells and under conditions described in (M). (M) Secreted collagen levels quantified by picro sirius red staining in extracellular matrix (ECM) derived from MEFs with sgCtrl, sgNadk2-1, or sgNadk2-2, cultured for 48 hours in DMEM or DMEM supplemented with $300 \mu \mathrm{M}$ proline, in the presence of $50 \mu \mathrm{M}$ ascorbate. (N) Pearson correlation of NADK2 mRNA level and forced vital capacity (FVC) before bronchodilator (pre-BD) as percentage of what was predicted for each patient. Data from GSE32537. (O) Pearson correlation of NADK2 mRNA level and diffusing capacity for carbon monoxide (DLCO) as percentage of what was predicted for each patient. Data from GSE32537. Error bars in (E) represent mean $\pm S D, n=10$. All other error bars in this figure represent mean + $\mathrm{SD}, \mathrm{n}=3$. In (B to $\mathrm{D})$, one-way ANOVA was performed. In $(E)$ and $(M)$, two-sided $t$-test was performed with Welch's correction. ${ }^{*} P<0.05$; ${ }^{* *} P<0.01$; ${ }^{* *} P<0.001$. 